\title{
The optical spectrum of the Vela pulsar ${ }^{\star}$
}

\author{
R. P. Mignani ${ }^{1}$, S. Zharikov ${ }^{2}$, and P. A. Caraveo ${ }^{3}$ \\ 1 Mullard Space Science Laboratory, University College London, Holmbury St. Mary, Dorking, Surrey, RH5 6NT, UK \\ e-mail: rm2@mssl.ucl.ac.uk \\ 2 Instituto de Astronomía, Universidad Nacional Autónoma de México, Apartado Postal 877, 22830, Ensenada, \\ Baja California, México \\ e-mail: zhar@astrosen.unam.mx \\ 3 INAF, Istitituto di Astrofisica Spaziale, via Bassini 15, Milan 20133, Italy \\ e-mail: pat@iasf-milano.inaf.it
}

Received 2 May 2007 / Accepted 8 July 2007

ABSTRACT

\begin{abstract}
Context. Our knowledge of the optical spectra of Isolated Neutron Stars (INSs) is limited by their intrinsic faintness. Among the fourteen optically identified INSs, medium resolution spectra have been obtained only for a handful of objects. No spectrum has been published yet for the Vela pulsar (PSR B0833-45), the third brightest $(V=23.6)$ INS with an optical counterpart. Optical multi-band photometry underlines a flat continuum.

Aims. In this work we present the first optical spectroscopy observations of the Vela pulsar, performed in the 4000-11 000 A spectral range.

Methods. Our observations have been performed at the ESO Very Large Telescope (VLT) using the FORS2 instrument.

Results. The spectrum of the Vela pulsar is characterized by a flat power-law $F_{v} \propto v^{-\alpha}$ with $\alpha=-0.04 \pm 0.04$ (4000-8000 $\mathrm{A}$ ), which compares well with the values obtained from broad-band photometry. This confirms, once more, that the optical emission of Vela is entirely of magnetospheric origin.

Conclusions. The comparison between the optical spectral indeces of rotation-powered INSs does not show evidence for a spectral evolution, suggesting that, as in the X-rays, the INS aging does not affect the spectral properties of the magnetospheric emission. At the same time, the optical spectral indeces are found to be nearly always flatter then the X-rays ones, clearly suggesting a general spectral turnover at lower energies.
\end{abstract}

Key words. techniques: spectroscopic - stars: pulsars: individual: PSR B0833-45

\section{Introduction}

The study of the optical emission properties of Isolated Neutron Stars (INSs) is hampered by their intrinsic faintness. For most of them the knowledge of the optical spectrum is still based on the comparison between multi-band photometry measurements. This comparison is affected by several uncertainties since photometry measurements are often compiled from the literature and, thus, are taken with different instruments and filters, calibrated with different photometric systems (167 used in total; see, e.g. Mono \& Munari $2000^{1}$ ), independently corrected for the atmospheric and interstellar extinction and converted to spectral fluxes using slightly different techniques. Only for five of the fourteen INSs with identified optical counterparts (see Mignani et al. 2004; Mignani 2005, for updated reviews) have mediumresolution spectra been obtained so far. The first one was the Crab pulsar (Oke 1969), the youngest ( 1000 years) and the brightest INS ( $V=16.6)$, for which repeated spectroscopy observations have been performed (Nasuti et al. 1996; Sollerman et al. 2000). The second one was PSR B0540-69 (Hill et al. 1997; Serafimovich et al. 2004), the youngest ( 2000 years) and brightest $(V=22.4$ ) INS after the Crab pulsar. Only after the advent of the $10 \mathrm{~m}$ class telescopes, has it been possible

\footnotetext{
* Based on observations collected at the European Southern Observatory, Paranal, Chile under programme ID 66.D-0261(A).

${ }^{1}$ http://ulisse.pd.astro.it/Astro/ADPS/
}

to obtain spectra of the fainter $(V \sim 25-25.6)$ and older ( $\geq 10^{5}$ years) INSs such as Geminga (Martin et al. 1998) and PSR B0656+14 (Zharikov et al. 2007), as well as of the radioquiet INS RX J1856-3754 (van Kerkwijk \& Kulkarni 2001).

The best next target for optical spectroscopy is the Vela Pulsar (PSR B0833-45), the optically third brightest ( $V=23.6$ ) INS. Among the first radio pulsars discovered in the sixties, its optical identification was proposed by Lasker (1976) and confirmed by the detection of optical pulsations at the radio period (Wallace et al. 1977). The high pulsed fraction of the optical lightcurve (see also Gouiffes 1998), suggested a non thermal, magnetospheric origin of the optical radiation. This was confirmed by multi-band photometry performed by Nasuti et al. (1997) with the NTT and by Mignani \& Caraveo (2001) with the HST. The fluxes between $\sim 3000 \AA$ and $\sim 8000 \AA$ follow a flattish, power-law distribution $\left(F_{v} \propto v^{-\alpha} ; \alpha=-0.2 \pm 0.2\right)$. The non-thermal nature of the optical radiation of the Vela pulsar has been confirmed by polarisation observations performed with the VLT (Wagner \& Seifert 2000; Mignani et al. 2007), pointing to a relatively high polarisation level. The Vela pulsar has been observed in the IR with the VLT (Shibanov et al. 2003), and in the near-UV (Romani et al. 2005; Kargaltsev \& Pavlov 2007) with the HST. While its multi-band photometry now extends from the IR to the near-UV, no optical spectrum has yet been published. Here, we report on the first spectroscopy observations of the Vela pulsar performed with the VLT. Observations, data analysis and 


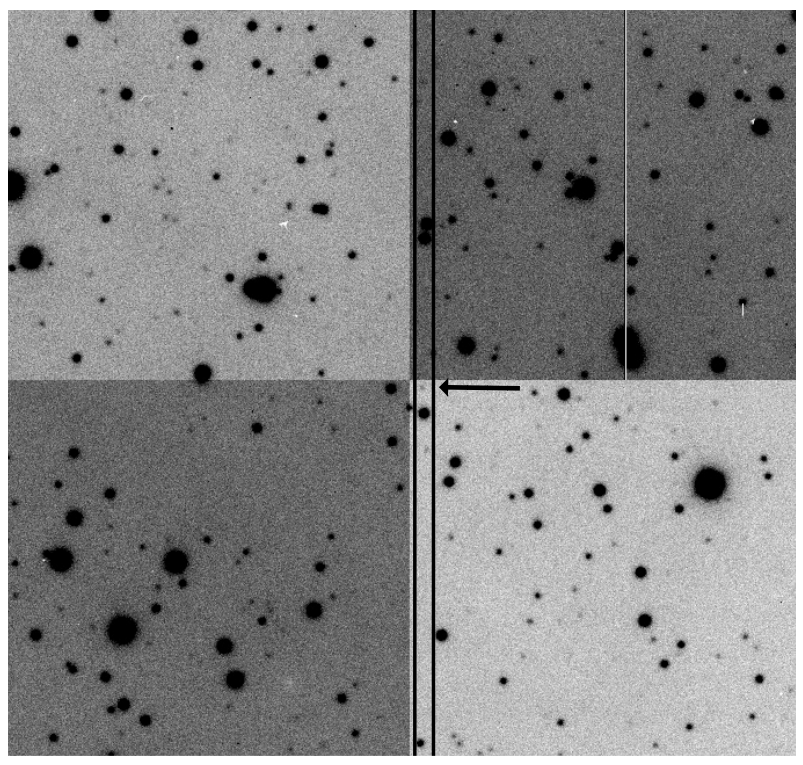

Fig. 1. VLT FORS2 raw $B$-band acquisition image of the Vela pulsar. North to the top, East to the left. The 2".5 LSS slit is overplotted, with the orientation NS as used in the first night (see Table 1). The position of the Vela pulsar (barely detected in the $60 \mathrm{~s}$ exposure acquisition image) is marked by the arrow. The difference between the four quadrants corresponds to the different CCD readout ports.

results are described in Sect. 2, and the interpretations are discussed in Sect. 3.

\section{Observations and data analysis}

\subsection{Observations}

Spectroscopy observations of the Vela pulsar were performed in Service Mode on four different nights between December 2000 and January 2001 with the VLT at the ESO's Paranal Observatory (Chile). We have used FORS2 (FOcal Reducer Spectrograph ${ }^{2}$ ), a multi-mode camera for imaging and long-slit/multi-object spectroscopy which is identical to FORS1 (Appenzeller et al. 1998) but with the CCD optmized to achieve a higher sensitivity in the Red part of the spectrum. At the time of the observations FORS2 was still equipped with the original detector, a Tektronix $2048 \times 2046$ pixels CCD with a plate scale of 0.20 with the Standard Resolution (SR) collimator. The instrument was operated in its single-port, high-gain, read out mode which is the default one for long slit spectroscopy (LSS). To cover the wavelength interval 4000-11000 ̊ the observations were performed with two different grisms: the $300 \mathrm{~V}(\Delta \lambda=4500-8600 \AA)$ and the $300 I$ one $(\Delta \lambda=6000-11100 \AA)$, equipped with the order separation filter $O G 590$. Both grisms have a dispersion of $\sim 2.7 \AA /$ pixel and a resolving power $\lambda / \Delta \lambda=440$. A slit width of 2.5 was used to collect as much flux as possible from the pulsar (see Fig. 1).

Single LSS science exposures of $2800 \mathrm{~s}$ each were obtained for both grisms and repeated for cosmic ray filtering. A total of eight $2800 \mathrm{~s}$ exposures were taken for a total integration time of $11200 \mathrm{~s}$ for each grism. The complete log of the science observations is reported in Table 1. The first exposure sequence in the $300 I$ grism was aborted and is not considered in the following analysis. The seeing conditions were always sub-arcsec

\footnotetext{
${ }^{2}$ www.eso.org/instruments/fors
}

Table 1. Summary of the spectroscopy observations of the Vela pulsar taken with FORS2. The columns give the observing dates, the used grism, the exposure times, the number of exposures per pointing $N$, and the average seeing and airmass values during each exposure sequence.

\begin{tabular}{|c|c|c|c|c|c|}
\hline yyyy-mm-dd & Grism & Time (s) & $N$ & Seeing & Airmass \\
\hline $2000-12-03$ & $300 \mathrm{~V}$ & 2800 & 2 & 0.79 & 1.14 \\
\hline $2001-01-23$ & $300 \mathrm{~V}$ & 2800 & 2 & 0.60 & 1.07 \\
\hline $2001-02-25$ & 300I & 1400 & 1 & 0.89 & 1.09 \\
\hline $2001-01-30$ & $300 \mathrm{I}$ & 2800 & 4 & 0.73 & 1.11 \\
\hline
\end{tabular}

( 0 ' 8 average) and the airmass below 1.2. The slit was oriented NS on the first night and EW on the following nights. For each night, daytime calibrations (biases, darks, flatfields, arc lamp spectra) were taken to correct for instrumental effects and to perform the wavelength calibration. Multi object spectroscopy (MOS) observations of the spectrophotometric standard stars LTT 3218, Feige 56 and Feige 67 (Hamuy et al. 1994) were acquired at the beginning of each night (with the exception of the third one) for flux calibration. Because of their brightness, standard stars were observed in MOS mode (slitlet size 22'.0) to avoid flux losses which might occur when using the narrower LSS slit (2'.5). Thanks to the sub-arcsec seeing conditions during the observations it was possible to resolve the pulsar spectrum in each individual science exposure. As an example, we show in Fig. 2 one of the two $300 \mathrm{~V}$ spectra taken on the first night, where the pulsar is clearly detected. Unfortunately, the pulsar is detected with a much lower significance in the $300 I$ spectra due to the higher sky background and to the decrease of the CCD sensitivity towards longer wavelengths

\subsection{Data analysis}

Spectral data reduction and calibration were performed using standard suites of tools for treating CCD data available in the MIDAS and IRAF sofware packages. Master calibration frames (master biases and darks, flux-normalized flat fields) were provided by the FORS data reduction pipeline ${ }^{3}$. Science and standard star spectra, as well as arc lamp spectra, were then bias and dark-subtracted, and flatfielded. Wavelength calibration was computed from the reduced arc lamp spectra by fitting a second order polynomial, yielding an rms of $0.4 \AA /$ pixel. The wavelength calibration was then applied row-by-row to both the standard stars and science spectra. For each grism, the flux normalization was computed from the extracted one-dimensional standard star spectrum using the available flux reference table and applying the atmospheric extinction correction with the extinction curves measured for the Paranal Observatory (Patat 2004). For each grism, single science spectra were finally coadded and cosmic rays removed. The pulsar one-dimensional spectrum was then extracted from each of the co-added science spectra using a 4 pixel wide $\left(0{ }^{\prime} 8\right)$ extraction window centered on the pulsar position where the $S / N$ is higher. The sky background was computed and subtracted from two 6 pixel wide (1.'2) regions immediately adjacent to the pulsar spectrum. For each extracted one-dimensional spectrum of the pulsar we applied a flux correction factor, computed from the extracted spectra of brighter field stars, to account for the width difference between the extraction window and the object's PSF. The spectra were corrected for the interstellar extinction using as a reference $E(B-V)=0.05$ obtained from the spectral fit to the XMM X-ray spectrum (e.g. Manzali et al. 2007). The two one-dimensional

\footnotetext{
${ }^{3}$ http://www.eso.org/observing/dfo/quality/FORS1
} 


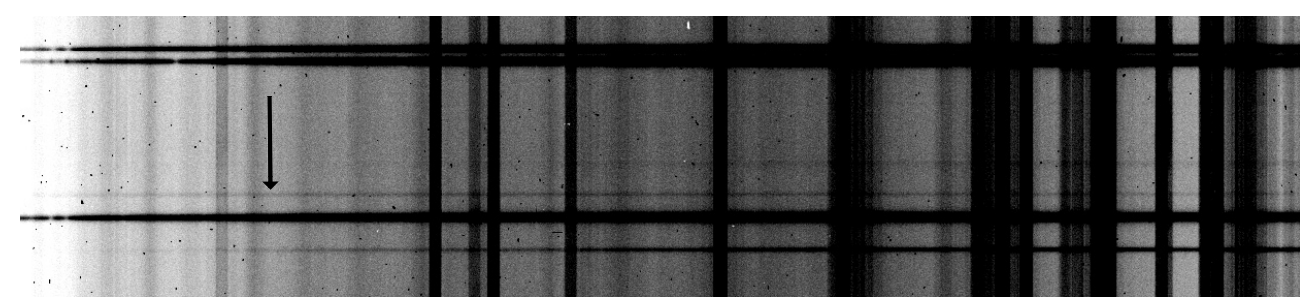

Fig. 2. VLT/FORS2 two-dimensional spectrum (first night) taken through the $300 \mathrm{~V}$ grism and the $2{ }^{\prime \prime} 5$ slit oriented NS (see Fig. 1). The Vela pulsar spectrum is marked with the arrow.

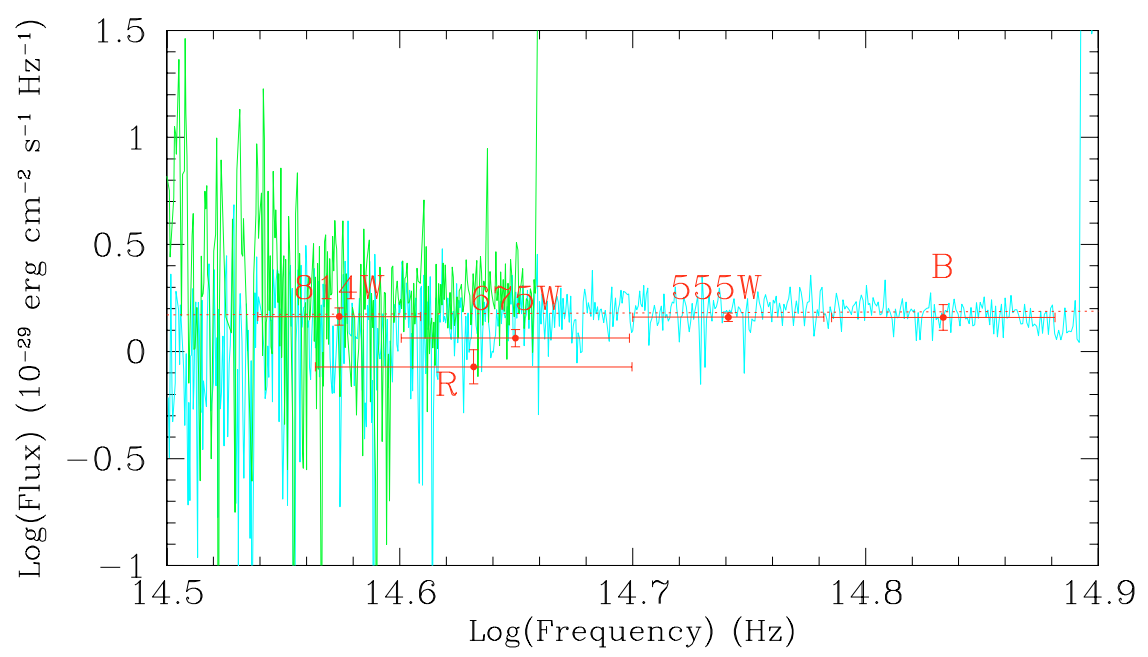

Fig. 3. Extracted one-dimensional spectrum of the Vela pulsar obtained by merging the $300 \mathrm{~V}$ and $300 \mathrm{I}$ spectra (shown in green and cyan, respectively) after wavelength/flux calibration, extinction correction, and coaddition. A rebinning of a factor 3 has been applied to both spectra. The spikes are due to defects in the subtraction of bright sky lines and of residual cosmic ray hits. The red points correspond to broad-band photometry (Nasuti et al. 1997; Mignani \& Caraveo 2001). The red dotted line represents the best fitting power-law $(\alpha=-0.04 \pm 0.04)$ to the continuum. Since at longer wavelengths the spectrum is dominated by the sky background, the fit has been performed only between 4000 and $8000 \AA$.

spectra of the pulsar were merged to yield a single spectrum across the 4000-11000 ̊ wavelength range.

\subsection{Results}

The final Vela pulsar spectrum is shown in Fig. 3. Due to the different detection significance of the pulsar in the two grisms, the $S / N$ of the resulting (unbinned) spectrum is rather unhomogeneous. In particular, we estimated a $S / N \sim 5$ (per pixel) in the 4000-5500 $\AA$ wavelength range and a $S / N$ as low as $\sim 1$ near/above $8000 \AA$. At longer wavelengths the spectrum is entirely dominated by noise. For this reason in the following analyses we have considered only the part of the spectrum between $4000 \AA$ and $8000 \AA$. Clearly, the spectrum consists of a pure flat continuum, with no evidence for emission or absorption feautures. For comparison, we have overplotted the spectral fluxes recomputed from the optical multi-band photometry (Nasuti et al. 1997; Mignani \& Caraveo 2001), obtained between $\sim 4000 \AA$ and $\sim 8000 \AA$. To be consistent, we have recorrected these fluxes for the same value of the interstellar extinction used in this work $\left(A_{\mathrm{V}}=0.16\right)$, while both Nasuti et al. (1997) and Mignani \& Caraveo (2001) have used $A_{\mathrm{V}}=0.4$. For the interstellar extinction correction we have used the coefficients of Fitzpatrick (1999). As seen, within the cross-calibration uncertainties, the multi-band photometry and the spectroscopy flux measurements are consistent. A putative dip at $6500 \AA$ was hinted at in the multi-band photometry data of Mignani \& Caraveo (2001). However, its existence was not confirmed by the VLT photometry of Shibanov et al. (2003). No evidence for such a dip is found in our spectral data either. We thus conclude that it was just the result of a cross-calibration problem in the multi-band photometry of Mignani \& Caraveo (2001).

We have fitted the FORS2 spectrum between 4000 and $8000 \AA$ with a power-law $\left(F_{v} \propto v^{-\alpha}\right)$ and we derived a spectral index $\alpha=-0.04 \pm 0.04$. This value supercedes the one obtained from the multi-band photometry of Mignani \& Caraveo (2001) on the basis of only five spectral flux measurements. We note that our spectral index is somewhat flatter than the one obtained by Shibanov et al. (2003) by fitting the IR-to-optical spectral fluxes $(\alpha=0.12 \pm 0.05)$, while it compares better with the spectral index $\alpha=0.01 \pm 0.02$, obtained by Kargaltsev \& Pavlov (2007) by extending the fit to the near-UV. Interestingly, the optical power-law is below the extrapolation of the power-law component $(\alpha=1.2 \pm 0.3)$ used to fit the XMM X-ray spectrum (Manzali et al. 2007; see also Kargaltsev \& Pavlov 2007), which clearly suggests that the magnetospheric emission of the Vela pulsar features a spectral turnover at longer wavelengths.

\section{Discussion}

We have compared the spectrum of the Vela pulsar with those of the other rotation-powered INSs for which either mediumresolution spectroscopy or multi-band photometry is available (Fig. 4). Apparently, the complexity of the spectral flux distribution grows with the INS age. For the young objects the optical spectral energy distribution is dominated by a flat power-law continuum which gives the signature of synchrotron 
Table 2. Summary of the measured optical/IR spectral index $\alpha_{\mathrm{O}}$ of rotation-powered INSs as obtained from spectroscopy (column three) and multi-band photometry (column five). Columns $2-4$ give (in logharitmic units) the pulsar spin down age $\tau$, the magnetic field $B$, and the rotational energy loss $\dot{E}$. The last column gives the phase-averaged X-ray spectral index $\alpha_{\mathrm{X}}$.

\begin{tabular}{|c|c|c|c|c|c|c|c|c|c|}
\hline Name & $\begin{array}{c}\log (\tau) \\
\text { yrs }\end{array}$ & $\begin{array}{c}\log (B) \\
(\mathrm{G})\end{array}$ & $\begin{array}{l}\log (E) \\
\left(\operatorname{erg~s}^{-1}\right) \\
\end{array}$ & $\alpha_{\mathrm{O}, \mathrm{sp}}$ & $\begin{array}{l}\lambda \lambda \\
(\AA) \\
\end{array}$ & $\alpha_{\mathrm{O}, \mathrm{ph}}$ & $\begin{array}{l}\lambda \lambda \\
(\AA)\end{array}$ & $\alpha_{\mathrm{X}}$ & Ref. \\
\hline Crab & 3.1 & 12.58 & 38.65 & $-0.11 \pm 0.04$ & $3300-9250$ & & & $1.079 \pm 0.004$ & 1,2 \\
\hline B1509-58 & 3.2 & 13.19 & 37.25 & & & 0.5 & $6000-18000$ & $0.4 \pm 0.5$ & 3,4 \\
\hline В0540-69 & 3.2 & 12.70 & 38.17 & $1.88 \pm 0.01$ & $3605-6060$ & $1.07 \pm 0.2$ & $3300-8000$ & $0.88 \pm 0.11$ & 5,4 \\
\hline Vela & 4.1 & 12.53 & 36.84 & $-0.04 \pm 0.04$ & $4000-8000$ & $0.01 \pm 0.02$ & $1500-16000$ & $1.2 \pm 0.3$ & $6,7,8$ \\
\hline B $0656+14$ & 5.0 & 12.67 & 34.58 & $0.2 \pm 0.2$ & $4600-7000$ & 0.41 & $4300-18000$ & $1.1 \pm 0.3$ & $9,10,11$ \\
\hline Geminga & 5.5 & 12.21 & 34.51 & $0.8 \pm 0.5$ & $3700-8000$ & 0.46 & $4300-16000$ & $0.7 \pm 0.1$ & $12,10,11$ \\
\hline B $1929+10$ & 6.5 & 11.71 & 33.59 & & & $0.5 \pm 0.5$ & $1700-3400$ & $1.7 \pm 0.1$ & 13,14 \\
\hline B0950+08 & 7.2 & 11.39 & 32.75 & & & $0.65 \pm 0.4$ & $3600-8000$ & $0.9 \pm 0.1$ & 15,16 \\
\hline
\end{tabular}

(1) Sollerman et al. (2000), (2) Kirsch et al. (2006), (3) Kaplan \& Moon (2006), (4) Gotthelf (2003), (5) Serafimovich et al. (2004), (6) this work, (7) Kargaltsev \& Pavlov (2007), (8) Manzali et al. (2007), (9) Zharikov et al. (2007), (10) Koptsevich et al. (2001), (11) De Luca et al. (2005), (12) Martin et al. (1998), (13) Mignani et al. (2002), (14) Becker et al. (2006), (15) Zharikov et al. (2004), (16) Becker et al. (2004).

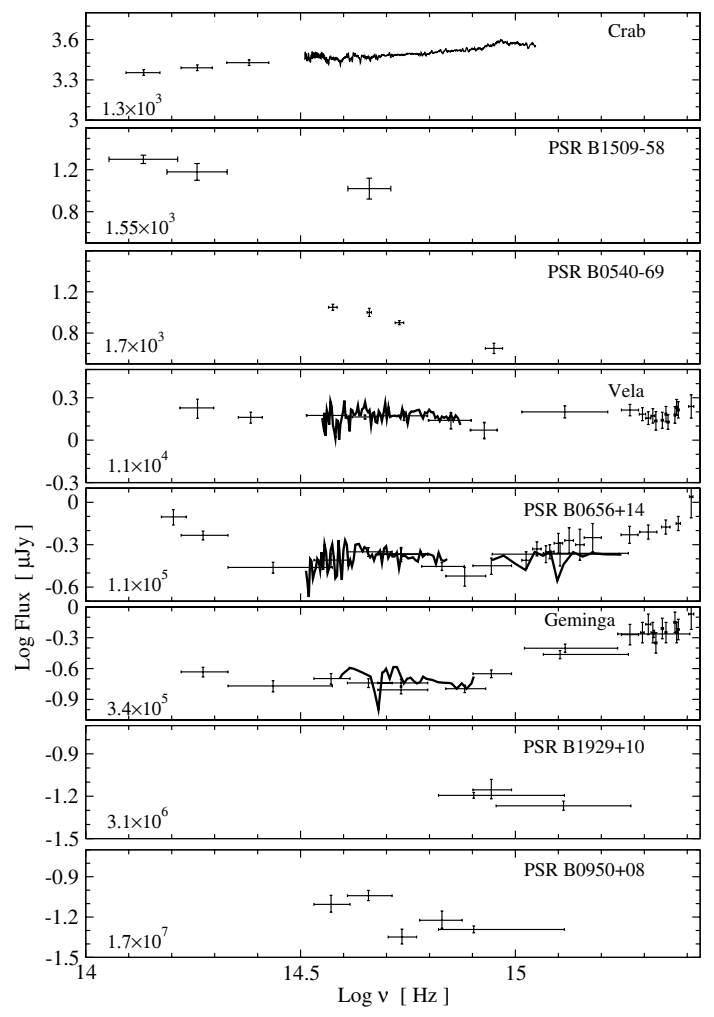

Fig. 4. Spectral flux distribution of all rotation-powered pulsars for which either medium-resolution spectroscopy or multi-band photometry is available (see Table 2). From top to bottom, objects are sorted according to increasing spin-down age. This figure has been updated from Fig. 1 of Zharikov et al. (2007), by including the IR fluxes of PSR B1509-58 (Kaplan \& Moon 2006), the Vela spectrum (this work), and the near-UV fluxes of Vela (Romani et al. 2005), Geminga (Kargaltsev et al. 2005) and PSR B0656+14 (Shibanov et al. 2005; Kargaltsev \& Pavlov 2007).

radiation produced by relativistic charged particles in the neutron star's magnetosphere. For the older ones, a Rayleigh-Jeans component, ascribed to thermal radiation from the cooling neutron star's surface, is also present. In all cases, no statistically significant evidence of emission or absorption features is found. For all the objects in Fig. 4, Table 2 reports the measured spectral index $\alpha_{\mathrm{O}}$ of the power-law component, either derived from spectroscopy or from broad-band photometry. In those cases where both values are available the agreement is rather good, with the only exception being PSR B0540-69. However, as discussed in Serafimovich et al. (2004), its spectrum is unrecoverably polluted by the background of the surrounding, bright compact $\left(\sim 4^{\prime \prime}\right)$ synchrotron nebula. The comparison of the optical spectral indeces shows that it is difficult to find clear spectral templates for different INS groups ${ }^{4}$. This is shown in Fig. 5 (top left panel), where we have plotted the optical spectral index $\alpha_{\mathrm{O}}$ as a function of the INS spin-down age. When available, we have assumed as a reference the spectral index obtained from spectroscopy, with the obvious exception of PSR B0540-69 (see above).

Although the spectral index value seems generally to correlate with the spin-down age, with $\alpha_{\mathrm{O}} \approx(0.12 \pm 0.04) \log (\tau)$, the errors are such that most of the points are also consistent with a costant distribution around the average value $\left\langle\alpha_{\mathrm{O}}\right\rangle=0.44 \pm 0.4$. Thus, there is no firm evidence for an evolution of the pulsars' optical spectral index over four age decades. This is similar to what is found in the X-rays, where the available data also suggest that the value of the spectral index $\alpha_{\mathrm{X}}$ does not depend on the spin-down age (see e.g. Becker \& Trümper 1997). A possible anti correlation between the optical spectral index and the rotational energy loss $\dot{E}$, with $\alpha_{\mathrm{O}} \approx(-0.07 \pm 0.02) \log (\dot{E})$, can also be recognized in the data (Fig. 5, top right panel). However, as before, the large errors do not allow us to draw any firm conclusion. No trend can be recognized between the optical spectral index and the magnetic field $B$ (Fig. 5, bottom left panel).

As it has been pointed out (e.g. Mignani et al. 2004; Serafimovich et al. 2004), the optical and X-ray magnetospheric emission of rotation-powered pulsars are almost never described by the same spectral parameters. This is clearly shown in Fig. 5 (bottom right panel), where we have plotted the optical spectral index $\alpha_{\mathrm{O}}$ vs. the X-ray one $\alpha_{\mathrm{X}}$. As seen, the optical power-law index is often less steep than the X-ray one, suggesting that the spectra of rotation-power pulsars undergo a general turnover at lower energies. In particular, for the Crab pulsar the slope of the power-law changes from positive to negative when passing from the X-ray to the optical/IR domain, underlying an even more marked spectral turnover. Furthermore, no general positive or negative correlation is found between $\alpha_{\mathrm{O}}$ and $\alpha_{\mathrm{X}}$, which indicates that the X-ray and optical magnetospheric emission, although likely produced by the same physical processes, are not directly correlated. This finding is particularly interesting in comparison with the strong correlation between the optical

\footnotetext{
4 The values of $\alpha_{\mathrm{O}}$ have been computed over slightly different wavelength ranges, which makes a direct comparison more uncertain.
} 

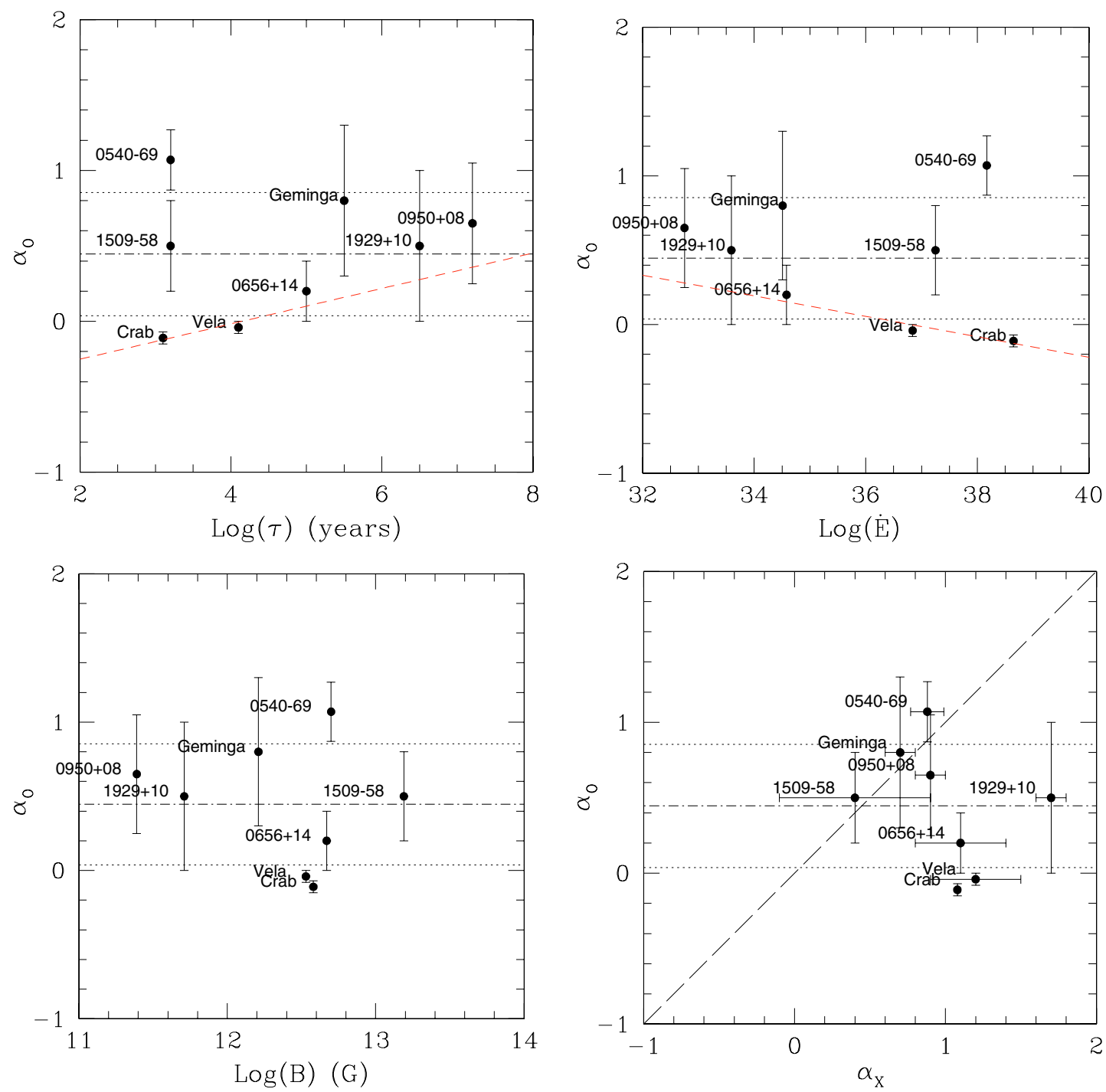

Fig. 5. Values of the optical spectral index $\alpha_{\mathrm{O}}$ plotted as a function of the INS spin-down age, rotational energy loss $\dot{E}$, the magnetic field $B$, and the $\mathrm{X}$-ray spectral index $\alpha_{\mathrm{X}}$ (top left to bottom right). The red dashed lines (top panels) represent the linear fit to the points. The dashed line (bottom right panel) marks the case $\alpha_{\mathrm{O}}=\alpha_{\mathrm{X}}$. In all panels the dot-dashed and dotted lines correspond to the average value $\left(\left\langle\alpha_{\mathrm{O}}\right\rangle=0.44 \pm 0.4\right)$ of the optical spectral index and to its $1 \sigma$ variation, respectively.

and X-ray luminosities of rotation-powered pulsars noticed by Zharikov et al. (2004) and by Zavlin \& Pavlov (2004).

\section{Summary}

We have presented the first optical spectroscopy observations of the Vela pulsar. The pulsar's spectrum is characterized by a flat power-law with spectral index $\alpha=-0.04 \pm 0.04$, consistent with the values derived from broad-band photometry (Mignani \& Caraveo 2001; Shibanov et al. 2003; Kargaltsev \& Pavlov 2007). We have compared the newly derived optical spectral index of Vela with those of all rotation-powered INSs for which a power-law component has been identified in the optical/IR spectrum. While a trend can be recognized in the data, the large errors in the spectral index values for most of the objects prevent any claim for an evolution of the magnetospheric emission properties over four age decades. We also found no evidence for a correlation between the optical and X-ray spectral indeces, which indicates that the $\mathrm{X}$-ray and optical magnetospheric emissions are not directly correlated. However, we showed that in the majority of cases, the optical spectral index is flatter than the
X-ray one, suggesting a turnover in the INSs' spectra at low energies.

Acknowledgements. R.P.M. is supported by a PPARC Rolling Grant. S.Z. acknowledges the support of the DGAPA/PAPIIT project IN101506 and of CONACYT 48493. We thank Werner Becker for the useful discussions and Oleg Kargaltsev for sending us the STIS/FUV pulsar fluxes. We thank our referee, Yury Shibanov, for his comments which helped to improve the quality of the manuscript.

\section{References}

Appenzeller, I., Fricke, K., Furtig, W., et al. 1998, The Messenger, 94, 1 Becker, W., \& Trümper, J. 1997, A\&A, 326, 682

Becker, W., Weisskopf, M. C., Tennant, A. F., et al. 2004, ApJ, 615, 908 Becker, W., Kramer, M., Jessner, A., et al. 2006, ApJ, 645, 1421

De Luca, A., Caraveo, P. A., Mereghetti, S., Negroni, M., \& Bignami, G. F. 2005, ApJ, 623, 1051

Fitzpatrick, E. L. 1999, PASP, 111, 63

Gouiffes, C. 1998, Proc. of Neutron Stars and Pulsars: Thirty years after the discovery, ed. N. Shibazaki et al. (Universal Academic Press), Frontiers Sci. Ser., 24, 363

Gotthelf, E. 2003, ApJ, 591, 361

Hamuy, M., Suntzeff, N. B., Heathcote, S. R., et al. 1994, PASP, 106, 566 
Hill, R. J., Dolan, J. F., Bless, R. C., et al. 1997, ApJ, 486, L99

Kaplan, D. L., \& Moon, D. 2006, ApJ, 644, 1056

Kargaltsev, O., \& Pavlov, G. G. 2007, Ap\&SS, 308, 287

Kargaltsev, O. Y., Pavlov, G. G., Zavlin, V. E., \& Romani, R. W. 2005, ApJ, 625, 307

Kirsch, M. G. F., Scoönherr, G., Kendziorra, E., et al. 2006, A\&A, 453, 173

Koptsevich, A. B., Pavlov, G. G., Zharikov, S. V., et al. 2001, A\&A 370, 1004

Lasker, B. 1976, ApJ, 203, 193

Manzali, A., De Luca, A., \& Caraveo, P. A. 2007, ApJ, accepted [arXiv:0706.3194]

Martin, C., Halpern, J. P., Schiminovich, D., et al. 1998, ApJ, 494, L211

Mignani, R. P. 1998, Proc. of Neutron Stars and Pulsars: Thirty Years after the Discovery, ed. N. Shibazaki et al. (Universal Academy Press), Frontiers Sci. Ser., 24, 335

Mignani, R. P. 2005, Proc. of The Electromagnetic Spectrum of Neutron Stars, ed. A. Baykal et al. (Springer), 210, 133

Mignani, R. P., \& Caraveo, P. A. 2001, A\&A, 376, 213

Mignani, R. P., De Luca, A., Caraveo, P. A., \& Becker, W. 2002, ApJ, 580, L147 Mignani, R. P., De Luca, A., \& Caraveo, P. A. 2004, Proc. of Young Neutron Stars and Their Environments, ed. F. Camilo, \& B. Gaensler, IAU Symp., 218, ASP Conf. Proc., 391

Mignani, R. P., Bagnulo, S., Dyks, J., Lo Curto, G., \& Słowikowska, A. 2007, A\&A, 467, 1157

Moro, D., \& Munari, U. 2000, A\&AS, 147, 361
Nasuti, F. P., Mignani, R., Caraveo, P. A., \& Bignami, G. F. 1996, A\&A, 314, 849

Nasuti, F. P., Mignani, R., Caraveo, P. A., \& Bignami, G. F. 1997, A\&A, 323, 839

Oke, J. B. 1969, ApJ, 156, L49

Patat, F. 2004, The Messenger, 118, 11

Romani, R. W., Kargaltsev, O., \& Pavlov, G. G. 2005, ApJ, 627, 383

Serafimovich, N. I., Shibanov, Y. A., Lundqvist, P., \& Sollerman, J. 2004, A\&A, 425,1041

Shibanov, Y. A., Koptsevich, A. B., Sollerman, J., \& Lundqvist, P. 2003, A\&A, 406, 645

Shibanov, Yu. A., Sollerman, J., Lundqvist, P., Gull, T., \& Lindler, D. 2005, A\&A, 440, 693

Sollerman, J., Lundqvist, P., Lindler, D., et al. 2000, ApJ, 537, 861

Wagner, S. J., \& Seifert, W. 2000, Proc. of Pulsar Astronomy - 2000 and Beyond, ed. M. Kramer et al., ASP Conf. Ser., 202, 315

Wallace, P. T., Peterson, B. A., Murdin, P. G., et al. 1977, Nature, 266, 692

van Kerkwijk, M. H., \& Kulkarni, S. R. 2001, A\&A, 378, 986

Zavlin, V. E., \& Pavlov, G. G. 2004, ApJ, 616, 452

Zharikov, S. V., Shibanov, Yu. A., Mennickent, R. E., et al. 2004, A\&A, 417, 1017

Zharikov, S., Mennickent, R. E., Shibanov, Y., \& Komarova, V. 2007, Ap\&SS, 308, 545 